\title{
StIgMa: Status Information Management for Evolvable Wireless Sensor Networks
}

\author{
Klaas Thoelen, Danny Hughes, Sam Michiels, Wouter Joosen \\ iMinds - DistriNet, KU Leuven, 3001 Leuven, Belgium \\ Email: first.last@cs.kuleuven.be
}

\begin{abstract}
The application of run-time evolvable software stacks promises an increase in the lifetime of wireless sensor network deployments. Besides supporting changing application requirements, said systems allow the underlying platforms to adapt to new environments by replacing system and communication services at runtime. These dynamics across various software layers however complicate the provision of accurate information about the current software and environmental status of a sensor node. This is problematic as sensor network management requires such information to configure efficient systems. As such, a mechanism needs to be in place via which run-time deployable software services can make their status information available to others in a standardized manner. In this paper we introduce StIgMa; a design for a unified node-local status registry on a sensor node. StIgMa's interfaces are cross-cutting and provide access to status information on all software layers on a sensor node. This is achieved without duplication of status data and avoiding tight coupling of status information providers and clients. To allow multi-user and flexible operation, StIgMa is supported by an extensible namespace for status parameters and a query language. Evaluation of a proof-of-concept implementation confirms that node-local centralized management of status information can be realized in a light-weight manner.
\end{abstract}

\section{INTRODUCTION}

Early research on wireless sensor networks (WSNs) primarily focused on single application deployments using dedicated, highly optimized and fixed software images deployed on sensor nodes in the field [1], [2]. Resource efficiency was achieved through optimization, however at the expense of evolution. Later, and current, research also investigates the applicability of evolvable WSN deployments; i.e. deployments in which the software stacks of individual sensor nodes evolve during their lifetime. Evolution is herein allowed not only at the application level [3], [4], but also at underlying system and communication levels [5]. This promises a longer lifetime of the deployed systems [6], which can now adapt to both new environments and application requirements. Optimization of resource use in said systems is to be provided by fine-tuning the current set of software services that are active on a sensor node at a given point in time.

In support of such fine-grained configuration, the current status of sensor nodes needs to be reifiable. This status can be interpreted as the set of information that describes currently deployed software services together with their configurable and evolving parameters, as well as parameters influenced by the environment and operation of a sensor node (e.g. current temperature and position, remaining energy level and memory). Such status information allows to target and adapt management actions by taking into account the current situation in which a sensor node finds itself. Examples are node and service discovery, group communication, resource management and policy reasoning. For instance, by specifying the wanted values of certain status parameters, a selection of sensor nodes can be described that form a group of nodes providing a certain service while also meeting a set of predefined conditions. More concretely, it allows us to identify one or more actuator sprinkler node(s) with the possibility of sensing temperature and enough free memory left to allow the deployment of an additional fire-detection service.

From the example given, it is clear that even individual management actions require status information that is scattered over various software layers and their constituent components. The presence of a sprinkler service is confirmed by an application-level service manager, while the presence of a hardware temperature sensor and the available memory is provided by a lower-level resource manager. This gives rise to two problems. Firstly, retrieving status information from various providers increases the dependencies of the status client, leading to so-called spaghetti code and limiting reuse. As such, to preserve architectural properties like layering, modularity and reusability, status sharing may not be implemented by tightly coupling status providers and clients through direct interface calls. Secondly, the functionality of retrieving status information and evaluating a node's current status is reusable itself and is better provided as a separate supporting middleware service available to a changing set of clients across multiple software layers.

Current state-of-the-art in WSN research acknowledges the benefits of incorporating status information into application and system management [7], [8], [9], [10], group communication [9], [11], [12] and service discovery [13], [14]. However, status information is mostly collected on an individual basis, not shared among various services and only provides a limited or compile-time defined set of parameters.

In this paper, we report on StIgMa; a design for a unified node-local status registry on a sensor node. It leverages on the classic use of a registry to centralize data; yet, in support of evolvable WSN platforms, we advocate its application as a generic middleware service that provides cross-layered interfaces for status provision and retrieval. Evolution is supported by an extensible namespace and a query language allows evaluation of more extensive status descriptions.

The rest of this paper is structured as follows. In Section II, 
we define what is understood by a sensor node's status. Section III outlines the requirements for managing status information on a sensor node and Section IV introduces an extensible namespace which covers all known status parameters in a WSN deployment. Section V describes the design of StIgMa, followed by a description of the query language it supports in Section VI. In Section VII we provide an extensive evaluation of an implementation before we discuss related work in Section VIII. We conclude in Section IX.

\section{A SEnsor Node's Status}

For the purposes of this paper, we define status as information contained in a software service on a sensor node that its owning entity is willing to share. These owning entities are termed status providers, and the software entities using this information are defined as clients. Note that both the provider and client role may be adopted by the same entity. The status information itself comprises hardware, software and environmental characteristics of a sensor node and may include the following:

- hardware characteristics: node brand and type, energy source, remaining energy, total memory, free memory, hardware sensors

- software characteristics: software names and versions, provided services and settings, service bindings, task description (e.g. identifier of product being monitored)

- environmental characteristics: current sensor values, position

This is an extensible set of parameters which are scattered over the various layers of the software stack. A number of these parameters, primarily those describing hardware characteristics and the characteristics of supporting software, will always be present in the status registry and are determined when the sensor node is prepared for deployment. Other parameters may be inserted and deleted during the node's lifetime. These mainly include the parameters of run-time deployable software services.

\section{REQUiREMENTS FOR STATUS INFORMATION MANAGEMENT}

Sharing of status parameters entails both collecting that information and offering it to clients. A status information management solution should thus satisfy the following set of functional requirements.

1) As status information needs to be shared across various layers of the software stack, a solution should cross-cut those layers.

2) The status data needs to be represented in a shared conceptualization which is extensible. This to support its multi-user application, and allow runtime addition and removal of status information in support of adaptation.

3) A solution should support both the retrieval of a single status parameter value as well as the evaluation of more elaborate status descriptions.
Additionally, in support of the adaptive and resource constrained environment, the following non-functional requirements should be taken into account.

1) Status providers and clients need to be decoupled in order to reduce explicit dependencies.

2) Status providers need to remain in control of their status data, preventing clients or other software entities from changing their status.

3) Redundancy of data, in the form of copies, needs to be avoided to conserve memory use.

4) Status parameters and descriptions need to be represented in a compact format to allow efficient transmission and processing of status information.

In the following sections we introduce our solution to this set of requirements. We first introduce an extensible namespace of status parameters and its distribution over the back-end and WSN. Afterwards, we discuss the design of StIgMa, a cross-layered registry that enables sharing of status information. Lastly, we describe the query language provided to retrieve status information using the shared conceptualization.

\section{Distribution OF INFORMATION}

A shared conceptualization of the status of sensor nodes is required in the multi-user environment under scope. This multi-user requirement stems from the fact that status information is to be shared among software services in various layers of the software stack. As application-, middleware- and operating system software is typically provided by different developers, and network managers need to be able to retrieve these services' status, an agreement needs to be in place concerning the identification of the status parameters and their processing.

We provide this shared conceptualization in the form of a namespace containing all currently used status parameters in the WSN deployment under scope. The following sections discuss how this namespace is fully described in the backend and only partially copied in the WSN as required for its correct operation.

\section{A. Back-end Status Representation}

The back-end status representation provides two lists specifying (i) the status parameter namespace and (ii) the supported data types for the these parameters.

The namespace represents an extensible list of all currently known status parameters. In a similar way to file systems and directory services, it is organized in a tree structure (see Figure 1), providing an adequate way to organize node status parameters in support of adaptation. Using its hierarchy of elements and the notion of sub-trees, data can be conveniently grouped together. As such, internal nodes represent a set of related status parameters; e.g. the energy node groups all energy related parameters of a node. While internal nodes often refer to a specific software service with its leaf nodes representing the affiliated status parameters, internal nodes might also be virtual (i.e. not linked to a status provider) with 
the intent of improving the structure of the tree. The provided tree structure furthermore allows for fast retrieval of data by hierarchical search.

The shared conceptualization also provides a limited list of data types that can be used to represent status data. For each status parameter, a specific data type is chosen, allowing clients to retrieve and use status information in the correct format.

As shown in Figure 1, each node in the tree is identified by a human-readable name and an encoding. Additionally a data type is specified. Encoding is performed in a single byte format, allowing up to 256 child nodes per parent node. The light status parameter is thus uniquely identified by /node/sensors/light, which in encoded form becomes $1 / 3 / 2$. The goal of the encoding is to reduce the system's overhead in three ways; communication, memory and processing. As sensor nodes are not user-centric devices (no visual display etc.) they require a more resource intensive back-end to allow interaction. As such, memory and communication overhead can be reduced by replacing human-readable strings as parameter identifiers by single-byte identifiers to store information on sensor nodes (see Section IV-B) and exchange status descriptions. For userfriendly interaction, back-end tools can resolve the numerical encodings to the human-readable names. Furthermore, traversing the status tree can be performed more efficiently in terms of processing when it involves single-byte comparisons instead of string comparisons.

Both the tree structure and its numerical encoding supports flexible addition of new elements to the tree. To every node in the tree, up to 256 child nodes can be added. As such, entire new sub-trees can be introduced into the hierarchy, representing the status of e.g. new software services. To avoid encoding inconsistencies, we adopt an append-only approach to the contents of the tree. Effectively, this means that encodings can only be used once and that even when their respective node is removed it cannot be reused. This is needed as the encoding might still be in use inside the WSN, referring to the old or removed status parameter. In extreme cases, it is possible to clean-up or re-encode the tree, yet this requires a full re-installation of the WSN deployments making use of that tree's namespace.

Finally, this shared conceptualization provides a two-fold abstraction of underlying operating systems, networking stacks and hardware. On the one hand does the specification of data types at the middleware level allow the description of status information in a platform independent manner. This enables the exchange of status information among services deployed on different platforms (i.e. different hardware, operating systems, etc.). On the other hand, while operating systems like Contiki [15] and TinyOS [1] have implementations for various hardware platforms, retrieving sensor data is often still done using hardware dependent API's, especially in case of external sensors. Or in the case of networking; e.g. neighbour tables are provided by different routing protocols through their specific interfaces. The status interface hides these platform and implementation differences behind a common interface

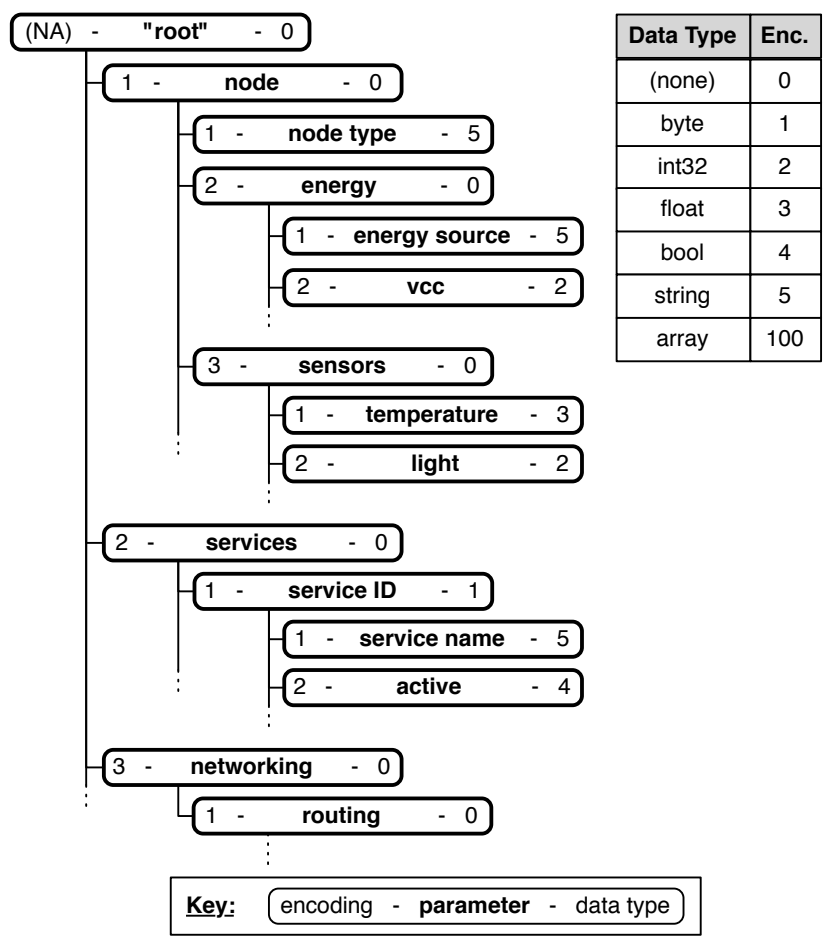

Fig. 1. A partial view on the back-end representation of the namespace describing all known status parameters in a deployment together with the encoding table for data types.

and naming system. As such, lower-level status data can be retrieved using standardized names; allowing more generic and reusable applications and services to be developed.

\section{B. On-node Status Representation}

While the back-end representation provides the full namespace of status parameters, the on-node representation can be regarded as a run-time instance of that namespace. Every deployed node maintains an individual data tree comprised of the status providers actually available on that node (see Figure 2). The list of status parameters shared by each provider are in turn maintained inside the provider itself. This limits the size of the on-node status tree to a minimum, consequentially minimizing memory use. Its individual elements reflect the encoding as used at the back-end, but omit the human-readable name and data type specification. The former can be omitted as the sensor node is not user-centric, while the data type specification can be retrieved from the back-end representation at development time and will not change at run-time. In contrast, where applicable, a pointer to a respective status provider is added to where the actual values of the respective status parameters are available (see Section V).

In contrast to the back-end representation, not only addition but also removal of status parameters at run-time is possible in the on-node representation. Both are the responsibility of the software services providing the respective status information or their managing entities. 


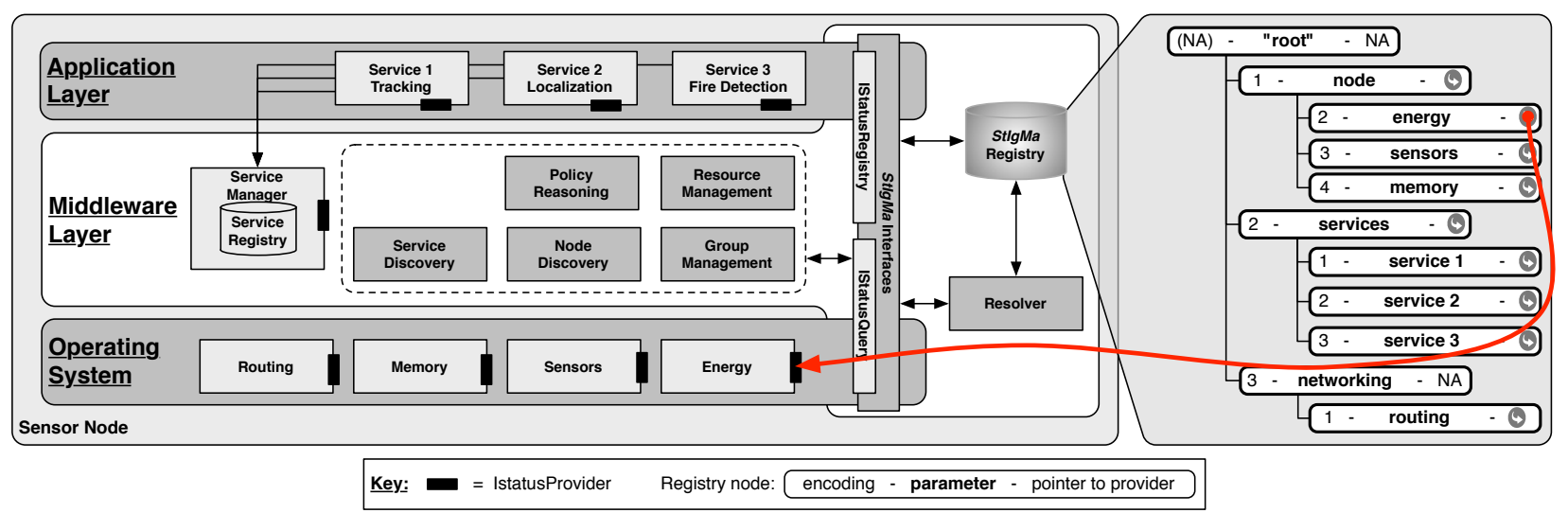

Fig. 2. The on-node StIgMa registry with status providers scattered across the service-based software stack. For clarity reasons only middleware services are shown as status clients, while this could potentially be any software service. The freehand arrow indicates an example of a pointer to a status provider.

\section{Node-local Status Registry Architecture}

The requirements of sharing status information across software layers and decoupling status providers and clients demand a central point in the software stack, which can be accessed by, and thus connects, various status providers and clients. To provide such a solution we draw inspiration from the registry pattern [16]. While this pattern is often applied in object-oriented programming to store away objects for later reuse, we apply it in a component-based programming context to store cross-layered references to software components, i.e. status providers.

The StIgMa status registry, depicted in Figure 2, is a central entity whose interface cross-cuts all layers of the software stack. It maintains a data structure that represents a status tree as described in Section IV-B. Figure 3 provides a more detailed architectural view, indicating the various interfaces between clients, status providers and the registry itself.

StIgMa supports three types of interaction through dedicated interfaces; registration, status provisioning and status querying. To be incorporated in the status tree, status providers, or their controlling entity, register their IStatusProvider interface (See Listing 1) at the IStatusRegistry interface of the registry. This makes status parameters of individual providers available to the status registry, and hence its clients. The IStatusQuery interface allows clients to retrieve status data. The getStatusParameter-method allows for the retrieval of the current value of the specified parameter. This parameter is the byteencoded path of a status parameter; e.g. [1,4,2] for /node/memory/free_memory. The registerStatusListener-method provides periodic retrieval of a status parameter. Besides the parameter's

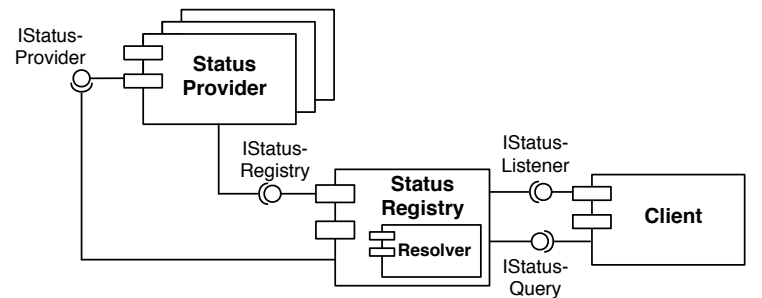

Fig. 3. An architectural view of StIgMa's status registry and its interfaces towards status providers and clients. byte-encoded path and a period, it requires the client to pass a reference to its implementation of the IStatusListener interface. Via this interface, updates of the status parameter are provided. When necessary, periodic retrieval can be stopped by using the unregisterStatusListener-method. The remaining two operations are used to evaluate predicates expressed in the query language discussed in Section VI. This evaluation is performed by the Resolver component. Either a simple boolean result can be retrieved via the boolResolve-method, or a specification of the actual status parameters via the resolvemethod.

In order to make their status information retrievable, status providers register themselves at the status registry. Knowing only an identification (i.e. the encoding) of a status parameter, clients then use the status registry to retrieve the matching value. In turn, the registry looks up the respective registered pointer to the status parameter, retrieves the value and passes it to the requesting client. By separating these interactions and implementing them through an intermediary status registry, we decouple status providers and clients. Additionally, the status registry retrieves the values of status parameters on-demand and does not duplicate status data. While the status parameters can thus be regarded as globally retrievable, we restrain access to them to only the status registry. Each status provider remains in control of its own data. Furthermore, such a central registry allows for easy and error-free addition and removal of status providers at run-time.

\section{The Query LANGUAGE}

Besides querying the registry for a single value, more extensive status descriptions can be passed to the Resolver as described in Section V. These descriptions take the form of an arbitrary logical expression using traditional relational operators $(<,>,=, \neq)$ and boolean logical operators $(\&, \mid)$; e.g. /node/node_type $=$ spot AND /node/memory/free $>3000$.

To reduce communication overhead when transmitting status descriptions and processing overhead during their evaluation, the logical expressions are encoded and formatted using reverse-polish notation [17]. Status parameters are encoded as described in Section IV-A and similarly a one byte encoding 


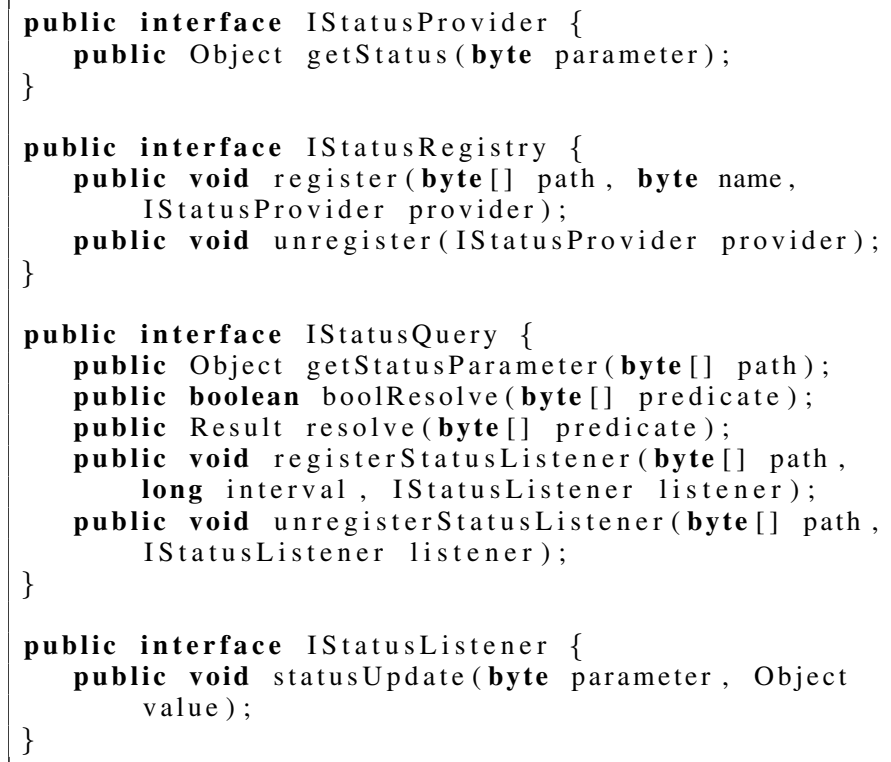

Listing 1. IStatusProvider, IStatusRegistry, IStatusQuery and IStatusListener interfaces.

is provided for the relational and logical operators. Encoded status descriptions thus take the form of a byte array, as is shown in Listing 3. The intention is however to generate these byte arrays from their more human-readable equivalents.

\section{IMPLEMENTATION AND EVALUATION}

In this section we evaluate StIgMa's design by means of an implementation that was realized for LooCI's Sun SPOT [3], [18] port. LooCI is the Loosely-coupled Component Infrastructure, which was selected as it allows for runtime adaptation of sensor nodes through deployment of application components. Sun SPOTs are relatively resource rich WSN devices (90MHz ARM920T 32 bit processor, 512KB RAM, 4MB Flash), which are programmable using Java ME. We evaluate this implementation of StIgMa in terms of memory overhead, processing overhead and programming overhead.

\section{A. Memory Overhead}

While we introduce an extra layer of indirection to retrieve status information, we have strived for a light-weight approach to minimize memory overhead. We evaluate both static and dynamic memory use and provide an overview in Table I. In the table, the first two lines are included to provide a reference for StIgMa's memory requirements. The first line presents the available amount of Flash and RAM memory on a Sun SPOT; the second line presents the Flash and RAM consumption of the LooCI Runtime implementation on Sun SPOT.

While the LooCI Runtime implementation can already be considered light-weight, including the status registry only consumes minimal extra memory; i.e. 20.9kB of Flash and 332 bytes of RAM. The relatively large Flash footprint is primarily caused by our implementation of the Resolver; on Sun SPOT each (empty) class increases Flash consumption with 230 bytes and a relatively large number of classes where needed to
TABLE I

MEMORY EVALUATION OF THE LOOCI SUN SPOT IMPLEMENTATION.

\begin{tabular}{|l|c|c|c|c|}
\hline & \multicolumn{2}{|c|}{ Flash (static) } & \multicolumn{2}{c|}{ RAM (dynamic) } \\
\hline \hline Sun SPOT (provided) & $4 \mathrm{MB}$ & $100 \%$ & $512 \mathrm{kB}$ & $100 \%$ \\
\hline LooCI Runtime & $34.9 \mathrm{kB}$ & $0.87 \%$ & $31.1 \mathrm{kB}$ & $6.1 \%$ \\
\hline \hline Status Registry & $20.9 \mathrm{kB}$ & $0.52 \%$ & $332 \mathrm{~B}$ & $0.065 \%$ \\
\hline Status Provider & $145 \mathrm{~B}$ & $0.004 \%$ & $17 \mathrm{~B}$ & $0.003 \%$ \\
\hline
\end{tabular}

represent the various operators and data types according to object-oriented principles. In contrast, dynamic memory of the status registry itself is low as it primarily provides a way to store references to status providers and therefore contains little state.

The last line in Table I, presents the memory overhead introduced per status provider. Transforming an existing software service into a status provider requires the implementation and registration of the IStatusProvider interface, which causes an increase of about 145 bytes of static memory use. This is depicted as well in Figure 4, which also shows the average increase of static memory use per status parameter provided. This is 8 bytes on average. Dynamic memory use increases with about 17 bytes per status provider, which is used to create a new node in the tree data structure including amongst others its encoded identification, the reference to the status provider itself and a list of its child nodes. This value does not change by the number of parameters provided by the status provider. This is because the tree data structure effectively only contains nodes for the status providers themselves and not for their status parameters. These parameters are already contained in the software service becoming a status provider and, by consequence, do not add additional dynamic memory overhead.

In conclusion, we can state that StIgMa's memory overhead is acceptable and are confident that its design can be implemented successfully on even more resource-constrained sensor nodes.

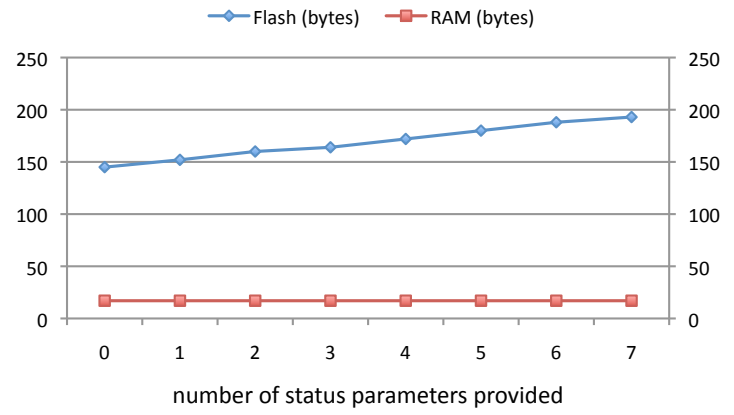

Fig. 4. Graph showing the static (Flash) and dynamic (RAM) memory use of a status provider with an increasing amount of status paramaters.

\section{B. Processing Overhead}

We evaluate the processing overhead of StIgMa's Sun SPOT implementation by means of time measurements of status retrieval and resolution of status expressions. With regards to status retrieval, we measured how long it takes to retrieve all 
status parameters of an extended version of the status tree as it is shown in Figure 1. On average this takes $7 \mathrm{~ms}$, with individual results ranging from less than a ms up to $29 \mathrm{~ms}$. The actual value depends on whether formatting of the result is needed at the status provider to fit in the generic Object that is returned by the getStatus and getStatusParameter methods.

The processing overhead of resolving logical expressions obviously depends on the size and complexity of the expression, as well as the properties of the parameters, which are part of this expression. The latter include their data type, depth in the tree and even their values themselves. As an indication, we measured the time it takes to evaluate a fairly complex expression; ((node/node_type $=$ spot OR nodelenergy/vcc $>$ 2500) AND (node/memory/free $>200000)$ ). On average, this is $87 \mathrm{~ms}$.

To conclude, we can state that, while subject to variability caused by the actual status information of interest, the processing overhead of StIgMa is acceptable.

\section{Programming Overhead}

In this last evaluation section, we discuss the programming overhead of status information provision and retrieval.

As described, provision of status information requires two actions; registration of the provider at StIgMa's registry and effectively providing access to the status parameters. As shown in Listing 2, registration of a status provider takes a single lineof-code to call the register method. Additionally, to provision status parameters, the IStatusProvider interface needs to be implemented. This interface provides only a single method, getStatus, which for every status parameter shared, returns the respective value. This is typically realized using a switch statement, resulting in about 2 lines-of-code per parameter. Depending on the number of parameters shared, the programming overhead of a status provider can thus be determined as a few lines-of-code up to a couple of tens of lines-of-code.

Retrieval of status information is performed by calling any of the methods of the IStatusQuery interface of the StIgMa registry as shown in Listings 1 and 3. In general, these calls require a single line-of-code, although additional programming effort is required for the creation of the query and retrieving a reference to the status repository itself. All in all, retrieval of

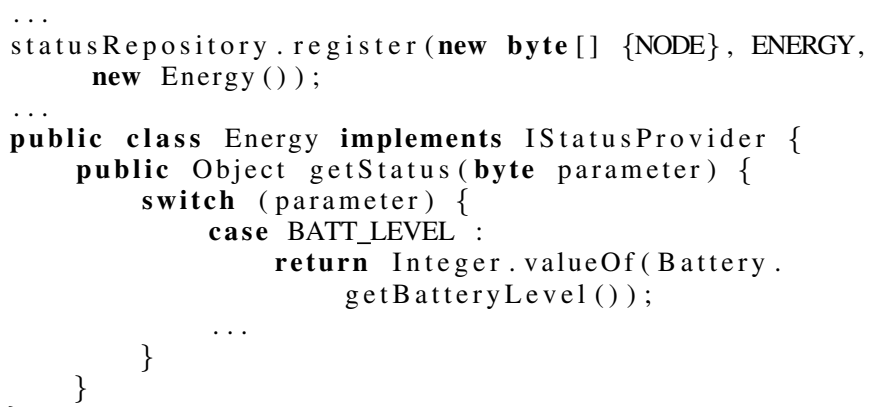

Listing 2. Status provisioning in the Sun SPOT implementation. a status parameter or evaluation of a logical status expression only requires a few lines of code.

We can thus state that programming overhead of status provision and retrieval is limited. Furthermore, we expect that most status providers will be middleware and system level software and thus application developers will only rarely register their applications as status providers. As is the case with LooCI, general component properties which are provided as status parameters, such as component name and a list of interfaces, are not registered by the application or component itself, which would require effort by the developer. Instead, the components' controlling entity within LooCI itself registers the components once they are deployed. This thus occurs without any action of the component's developer himself.

\section{RELATED WORK}

Our work is related to research on cross-layered interactions and data sharing in WSNs. In Chi [10], system configuration is separated from system logic by extracting configuration parameters of different system layers into a central registry, called a blackboard. Configuration policies operate on the configuration data on the blackboard to optimize the system. Although both approaches are similar, Chi enables centralized holistic configuration of all software layers, as reflected by its support for only integer-type parameters. We on the other hand, focus on cross-layered sharing of not only configuration parameters, but all status parameters considered relevant.

TinyXXL [19] provides language and runtime support for cross-layer data exchange. By using compile-time optimizations, it strives to ease the development of cross-layer optimized applications built from reusable components. In case adaptation is used, to allow at-runtime component deployment, not all optimizations are possible however. Furthermore, being an extension to nesC, TinyXXL heavily depends on TinyOS [1].

The COPAL [20] middleware allows context-aware services to subscribe and react to context changes. These changes are determined by the processing of events reported by a distributed set of publishers. Our scope is different in that

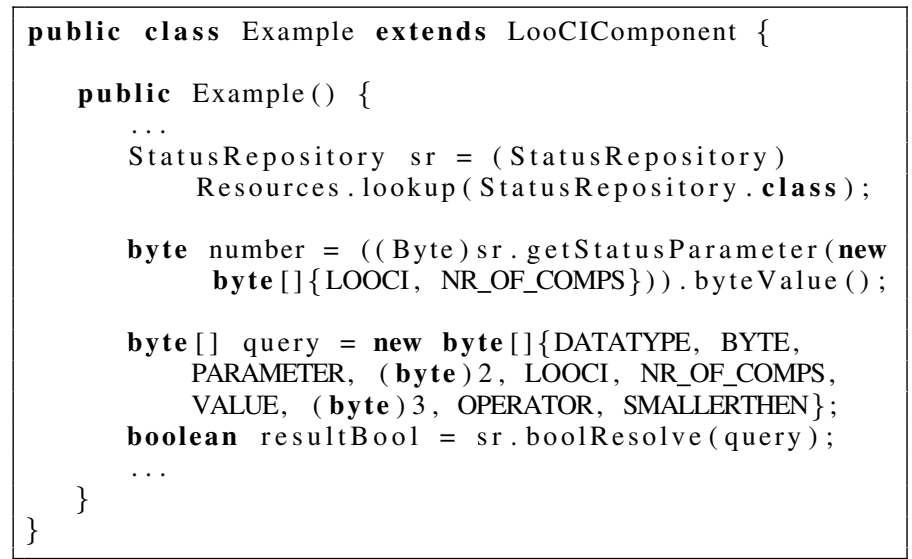

Listing 3. Status retrieval in the Sun SPOT implementation. 
we provide status information of a single node to support its management and applications, instead of processing that information into higher level context descriptions. Both approaches might however benefit of each other as COPAL might serve as both a status provider and client to the status registry.

In other research efforts, status information is used in a different scope of operations. In [11], the authors introduce a neighbourhood abstraction for WSNs called Logical Neighbourhoods. Node templates are used as an application level representation of the status of a node to determine group membership. The sets of parameters in these templates are determined at compilation time. The status registry described in this paper, however, allows more finer grained status provisioning by individual software services and allows run-time addition and removal of status providers and their respective status data.

Finally, tuple spaces for WSNs [12], [21] allow data sharing among neighbouring nodes via a programmatical interface which hides the distribution aspect. Data tuples are stored in the tuple space, after which they are disseminated to neighbouring nodes. We, however, focus on node-local sharing of status data and use an on-demand approach for data retrieval instead of proactive sharing of data.

\section{COnclusion \& Future Work}

In WSNs that support runtime deployment and reconfiguration of services on individual nodes, the status of those nodes becomes important to configure efficient systems and allow correct targeting of management actions to the most suitable nodes. In this paper, we proposed StIgMa, a node-local status registry that allows the sharing of status information across software layers without tightly coupling status providers and clients, and avoiding data duplication. The evaluation of our LooCI/Sun SPOT implementation has demonstrated that this can be realized in a memory, processing and programming efficient manner.

Our future work involves further improvement of the namespace and query language as described here. We are mainly interested in increasing the expressiveness of the query language and further incorporate it with management services like group management, service discovery and policy reasoning. Furthermore, we plan to implement StIgMa on more resourceconstrained sensor nodes to verify the light-weightiness of our solution and realize status sharing across various platforms.

\section{ACKNOWLEDGMENT}

This research is partially funded by the Interuniversity Attraction Poles Programme Belgian State, Belgian Science Policy, and by the Research Fund KU Leuven. It is conducted in the context of the IWT-SBO-STADiUM project [22].

\section{REFERENCES}

[1] "TinyOS," http://www.tinyos.net/, 2012.

[2] R. Szewczyk, J. Polastre, A. M. Mainwaring, and D. E. Culler, "Lessons from a sensor network expedition." in EWSN, 2004, pp. 307-322.
[3] D. Hughes, K. Thoelen, W. Horré, N. Matthys, P. J. del Cid Garcia, S. Michiels, C. Huygens, and W. Joosen, "Looci: A loosely-coupled component infrastructure for networked embedded systems," in Proceedings of the 7th Int. Conf. on Advances in Mobile Computing \& Multimedia. ACM, December 2009, pp. 195-203.

[4] C.-L. Fok, G.-C. Roman, and C. Lu, "Enhanced coordination in sensor networks through flexible service provisioning," in Proceedings of the 11th Int. Conf. on Coordination Models and Languages (COORDINATION). Berlin, Heidelberg: Springer-Verlag, 2009, pp. 66-85.

[5] B. Porter, U. Roedig, and G. Coulson, "Type-safe updating for modular wsn software," in Distributed Computing in Sensor Systems and Workshops (DCOSS), 2011 International Conference on, june 2011, pp. 1 -8 .

[6] A. Dearle and S. Dobson, "Mission-oriented middleware for sensordriven scientific systems," Journal of Internet Services and Applications, vol. 3, pp. 133-139, 2012.

[7] W. Horré, D. Hughes, S. Michiels, and W. Joosen, "Advanced sensor network software deployment using application-level quality goals," Journal of Software, vol. 6, no. 4, pp. 528-535, Apr. 2011.

[8] N. Matthys, C. Huygens, D. Hughes, J. Ueyama, S. Michiels, and W. Joosen, "Policy-driven tailoring of sensor networks," in Second International ICST Conference on Sensor Systems and Software ( $S$ CUBE), 2010, pp. 20-35

[9] L. Mottola, G. P. Picco, and A. A. Sheikh, "Figaro: Fine-grained software reconfiguration for wireless sensor networks." in Proceedings of the 5th European Conference on Wireless Sensor Networks (EWSN), 2008, pp. 286-304.

[10] N. Finne, J. Eriksson, N. Tsiftes, A. Dunkels, and T. Voigt, "Improving sensornet performance by separating system configuration from system logic," in Wireless Sensor Networks, ser. Lecture Notes in Computer Science. Springer Berlin / Heidelberg, 2010, vol. 5970, pp. 194-209.

[11] L. Mottola and G. P. Picco, "Logical neighborhoods: A programming abstraction for wireless sensor networks," in Proceedings of the 2nd Int. Conf. on Distributed Computing on Sensor Systems (DCOSS), 2006.

[12] K. Whitehouse, C. Sharp, E. Brewer, and D. Culler, "Hood: a neighborhood abstraction for sensor networks," in Proceedings of the 2nd Int. Conf. on Mobile Systems, applications, and services (MobiSys). New York, NY, USA: ACM, 2004, pp. 99-110.

[13] A. Rezgui and M. Eltoweissy, "Service-oriented sensor-actuator networks: Promises, challenges, and the road ahead," Computer Communications, vol. 30, pp. 2627-2648, September 2007.

[14] K. Thoelen, S. Michiels, and W. Joosen, "On-demand attribute-based service discovery for mobile wsans," in Proceedings of the 5th Int. Conf. on Communication System Software and Middleware, ser. COMSWARE '11. New York, NY, USA: ACM, 2011, pp. 7:1-7:6.

[15] “Contiki," http://www.contiki-os.org/, 2012.

[16] M. Grand, Java Enterprise Design Patterns: Patterns in Java, ser. Patterns in Java. John Wiley \& Sons, 2002.

[17] A. W. Burks, D. W. Warren, and J. B. Wright, "An analysis of a logical machine using parenthesis-free notation," Mathematical Tables and Other Aids to Computation, vol. 8, no. 46, pp. pp. 53-57, 1954.

[18] "Sun SPOT," http://www.sunspotworld.com/, 2012.

[19] A. Lachenmann, P. Marron, D. Minder, M. Gauger, O. Saukh, and K. Rothermel, "Tinyxxl: Language and runtime support for cross-layer interactions," in 3rd Annual IEEE Comm. Soc. on Sensor and Ad Hoc Communications and Networks (SECON), vol. 1, sept. 2006, pp. 178 $-187$.

[20] F. Li, S. Sehic, and S. Dustdar, "Copal: An adaptive approach to context provisioning," in 6th Int. IEEE Conf. on Wireless and Mobile Computing, Networking and Communications (WiMob), oct. 2010, pp. 286 -293.

[21] P. Costa, L. Mottola, A. L. Murphy, and G. P. Picco, "Teenylime: transiently shared tuple space middleware for wireless sensor networks," in Proceedings of the Int. Workshop on Middleware for Sensor networks (MidSens). New York, NY, USA: ACM, 2006, pp. 43-48.

[22] "IWT-STADiUM-SBO project 80037, Software Technology for Adaptable Distributed Middleware," http://distrinet.cs.kuleuven.be/projects/stadium/, 2011. 